Un marco basado en BIM para verificar la regla de circulación exterior

\section{| ABSTRACT |}

This paper introduces a BIM-based framework for outdoor circulation rule checking from a geometric modeling perspective. The paper extracts rules and patterns of circulation and interaction based on site planning standards and codes. It then identifies high-level operators and 3D low-level operations involved in applicable circulation rules, and suggest methods for implementation. Mechanisms of integration between high-level operators and low-level operations are defined for different domains of rule checking, based on three types of datasets: I) BIM objects in outdoor settings, including agents like pedestrians, bikes, vehicles, and other objects; 2) attributes and behavior of objects, and 3) interrelations among objects, including agent-agent or agent-object intersection conflicts, agent-agent or agent-object visual access, unobstructed access, and outdoor lighting and shading.

\section{RESUMEN}

Este artículo presenta un marco basado en BIM para la revisión de circulaciones exteriores desde una perspectiva de modelamiento geométrico. En el documento se extraen reglas y patrones de circulación e interacción basados en códigos y normas de planificación. A continuación se determinan operadores de alto nivel $y$ operaciones 3D de bajo nivel vinculados a reglas de circulación, sugiriendo métodos para su implementación. Definimos mecanismos de integración entre los operadores de alto nivel y las operaciones de bajo nivel para diferentes dominios de comprobación de reglas a partir de tres tipos de conjuntos de datos: I) objetos BIM en escenarios al aire libre, incluyendo agentes como los peatones, bicicletas, vehículos y otros objetos; 2) atributos y comportamiento de objetos; y 3 ) interrelaciones entre objetos incluyendo intersección de conflictos agenteagente o agente-objeto, accesibilidad visual, e iluminación y conos de sombra al aire libre.

\title{
A BIM-based framework for outdoor circulation rule checking
}

\section{INTRODUCTION}

The current trend in everyday design practice usually involves architects consulting building codes then submitting drawing sets to the specified building department that checks for compliance and typically returns them for revision until compliance is achieved, where the permit to construct is issued. The BIM-enabled version of this system shifts the code checking process to a rather automated one. Architects can use BIM software to present their designs, which are then imported into rule-based model checking software that return compliance reports to the architect or client. Many efforts have been done regarding automated rule-based checking in buildings, using building codes and design guides as compliance references (Ding et al., 2004; Eastman et al., 2009; Lee et al., 2010; Sanguinetti et al., 2012), relying on the Industry
Foundation Classes (IFC) open standard building model. Most rule checking systems work as a checking routine; i.e. after the main layout is generated, and not concurrently with the actual design to allow acceptable or non-acceptable designs. The focus is oriented toward checking special issues like space planning, circulation, building performance, in a variety of building types, such as courthouses, hospitals, schools, etc.

Efforts were also done regarding pedestrian simulation, urban flow modeling and space syntax (Hillier et al., 1993; Kerridge et al., 2001; Hoogendoorn, 2003; Raford and Ragland, 2003). Little has been done however in the area of outdoor circulation compliance and rule checking. Although most relationships and issues in outdoor settings involve a casual notion of interactions that may not require strict rule definitions as in buildings, an initial survey

Sherif M. Abdelmohsen is Assistant Professor of Architecture at Ain Shams University, Adjunct Faculty at the American University in Cairo (AUC), Research Manager at CORELAB, and founder of KRAFT|Studio, Cairo, Egypt. He holds a PhD in Architecture from Georgia Tech, USA in 2011 . He has also been involved in teaching and research at Georgia Tech, Carnegie Mellon University, British University in Egypt, Future University in Egypt, and Arab Academy for Science, Technology and Maritime Transport. His research lies at the intersection of design cognition and design computing, including building information modeling (BIM), parametric design, cognitive processes in design, sketch understanding, and responsive architecture. He has publications and presentations in Advanced Engineering Informatics, Automation in Construction, Architectural Design, CAADFutures, ACADIA, DCC, CAADRIA and IASDR. $\mathrm{He}$ is also a practising architect, freelance BIM consultant, member of Board of Directors for ASCAAD, and reviewer for Automation in Construction, eCAADe, CAAD Futures and ACADIA.

Department of Architecture, Faculty of Engineering, Ain Shams University 1 Sarayat St., Cairo, Egypt. Corresponding Author: Phone: +20222635025, +201227340487,Email: sherif.morad@gmail.com, Address: 14 Zaker Hussein St., Apt. 901, Nasr City, Cairo, Egypt 11471 
of design guides concerning site planning and layout design shows the contrary. This paper introduces a BIM-based framework for outdoor circulation rule checking from a geometric modeling standpoint. The paper identifies highlevel operators and low-level operations involved in applicable circulation rules, and suggests methods for implementation.

\section{APPROACH AND EXTRACTED CIRCULATION RULES}

There are some key points that distinguish outdoor from indoor circulation rules. First, the type of agents in each context and the nature of interaction among these agents is different. Within buildings, the focus is primarily on people as agents, moving both horizontally and vertically. In outdoor circulation however, the interaction between pedestrians, bikes and vehicles is a key issue. The nature of this interaction regulates most of the issues embedded in outdoor circulation rules. Second, the nature of outdoor circulation involves informal movement with fewer constraints than indoor circulation, which relies on specific start and target locations. For bikes and vehicles, there are often assigned routes for controlled movement, but for people, circulation is not really confined to specific nodes or start and end points, but more ad hoc. Circulation patterns tend to take the form of «allowed zones» of movement rather than specific routes.

Attributes of speed, density, flow and numbers of agents are also significant. In indoor circulation, specific elements like walls and spaces define the boundaries and zones of movement. Outdoor areas, however, are different. «Agent-specific» pathways (pedestrian walkways, bike lanes, roads) define the allowed zone of movement. In addition, other barriers and objects (fences, trees, bollards, benches, lighting poles) help define allowed circulation spaces. In indoor circulation, IFC development plays an important role in defining ready-to-implement elements, like walls, stairs, spaces, etc. IFC entities have not yet been fully into effect for site related objects like roads, paths, and paving.
To identify applicable circulation rules, the research looked at several site planning codes (Abbey, 1998; Harris \& Dines, 1998; Landphair \& Klatt, 1998; Ramsey, et al., 2000). The goal was to exhaust rules that could be implemented using a geometric modeling approach. It was necessary to identify three types of datasets: BIM objects, attributes, and inter-relations. BIM objects included main agents in outdoor contexts (pedestrians, bikes, and vehicles), in addition to objects like pedestrian walkway, bench, fence, bike driver, bike pathway, wheelchair, sign, underpass, overpass, lighting pole, bike rack, bike parking, vehicle driver, roadway lane, utility pole, fire hydrant, aerial ladder, kiosk, fountain, and parking lot.

Attributes were identified, like spatial bubbles, walking rates, walking distances, flow volume, horizontal and vertical cones of vision for pedestrians; speed, sight/stopping distance, and minimum curve radii for bikes; sightstopping distances, minimum crest vertical curves, and passing sight distance on vertical curves for vehicles. To define the inter-relationships between these objects, as implied in the rules, the paper focused on significant rule goals. Instead of focusing on just descriptions of dimensions of outdoor spaces, road widths, or walkway slopes, the focus was on rules that involved interaction between objects, especially:

1. Agent-agent / agent-object intersection conflict

e.g. «The purpose of bollards is to allow an unrestricted, barrier free flow of pedestrian, bicycle, and wheelchair traffic, while restricting the passage of such vehicles as cars and trucks». Ramsey et al. (2000).

2. Agent-agent / agent-object visual access e.g. «Signs erected at the roadside should be mounted with the lower edge of the sign no less than $5 \mathrm{ft}$ above the pavement on rural roadways, and $7 \mathrm{ft}$ above the pavement in residential, business and commercial districts and on expressways». Harris and Dines (1998).
3. Unobstructed Access e.g. «Utility poles can obstruct use of aerial ladders for rescue and fire suppression operations. Kiosks, outdoor sculpture, fountains, newspaper boxes, and the like can also seriously impede fire fighting operations». Ramsey et al. (2000).

4. Outdoor Lighting and Shading e.g. «Fixtures should provide an overlapping pattern of light at a height of about $7 \mathrm{ft}$. Posts and standards should be placed so that they do not create hazards for pedestrians or vehicles». Ramsey et al. (2000).

\section{HIGH-LEVEL OPERATORS}

To satisfy rule goals, it was necessary to define operators that could be used, individually or in conjunction, for checking the selected rules. These operators are defined as «high-level» operators, as they do not currently exist in model checking software, but entail operations that are domain-specific. Within the structure of each operator are low-level operations like Boolean, slicing and other geometric operations.

\section{CONSTRUCT ALLOWED CIRCULATION ZONE}

This operator defines the portion(s) of space permissible for agent circulation within the site. This is different for each type of agent. An important factor is the medium of circulation for each agent; pedestrian walkway, bike pathway, vehicle roadway, etc. The «allowed zone» defines the total usable circulation area. Usually bike/ vehicle circulation is more controlled than pedestrian circulation, where this allowed zone is an informal circulation area. This operator however attempts to identify all possible usable zones for pedestrian circulation. In other words, a representation of the flow, or sweep, of (a density of) maximum pedestrians in a specific area would define an allowed zone. It would then require the exclusion of all obstructing objects within the site. The allowed zone is represented by the «void» volume resulting from 
1. Generating "allowed circulation zone» of a pedestrian "agent» along a pedestrian walkway.

2. Generating «cone of vision» for a pedestrian «agent». the elimination of circulation zones for other agents, in addition to all obstructing BIM objects.

Active objects in this case include the main agents and their associated paths, e.g. pedestrian, pedestrian walkway, bike, bike pathway, vehicle, etc. Passive objects include benches, lighting poles, bollards, etc. Arguments for this operator include the type of agent, its circulation path coordinates, site coordinates of BIM object(s), bounding volume coordinates and dimensions for BIM object(s), and sweep height. This operator works as follows: 1) identifying the agent (pedestrian, bike, vehicle, etc.) for "allowed circulation», 2) generating coordinates of the area(s) of «allowed circulation» according to the associated circulation path (e.g. pedestrian $\Leftrightarrow$ pedestrian walkway), 3) generating an extrusion based on that area, taking the average agent height along the path as theextrusion height (FIGURE 1).

This operator applies to rules that mainly involve conflicts in agent/agent or agent/object intersection. Additional operators would be required to completely satisfy these rules, such as bounding volume and sweep operations. Low-level operations, like Boolean and other 3D editing operations, would be involved for the interaction among the high-level operators. The integration of these operators with the high-level operator should be capable of:

a. Identifying and enumerating all BIM objects that are «allowed» to intersect the path (e.g. trees in case of a pedestrian walkway), but where agents cannot physically access.

b. Subtracting these objects from the 3D extrusion volumes defined earlier (generated volumes represent allowed circulation zones for corresponding agent).

c. Identifying all objects that are not «allowed» to intersect the path (e.g. a bench in a continuous pedestrian flow).

\section{CONSTRUCT CONE OF VISION}

This operator defines capabilities in terms of visual access according to each individual
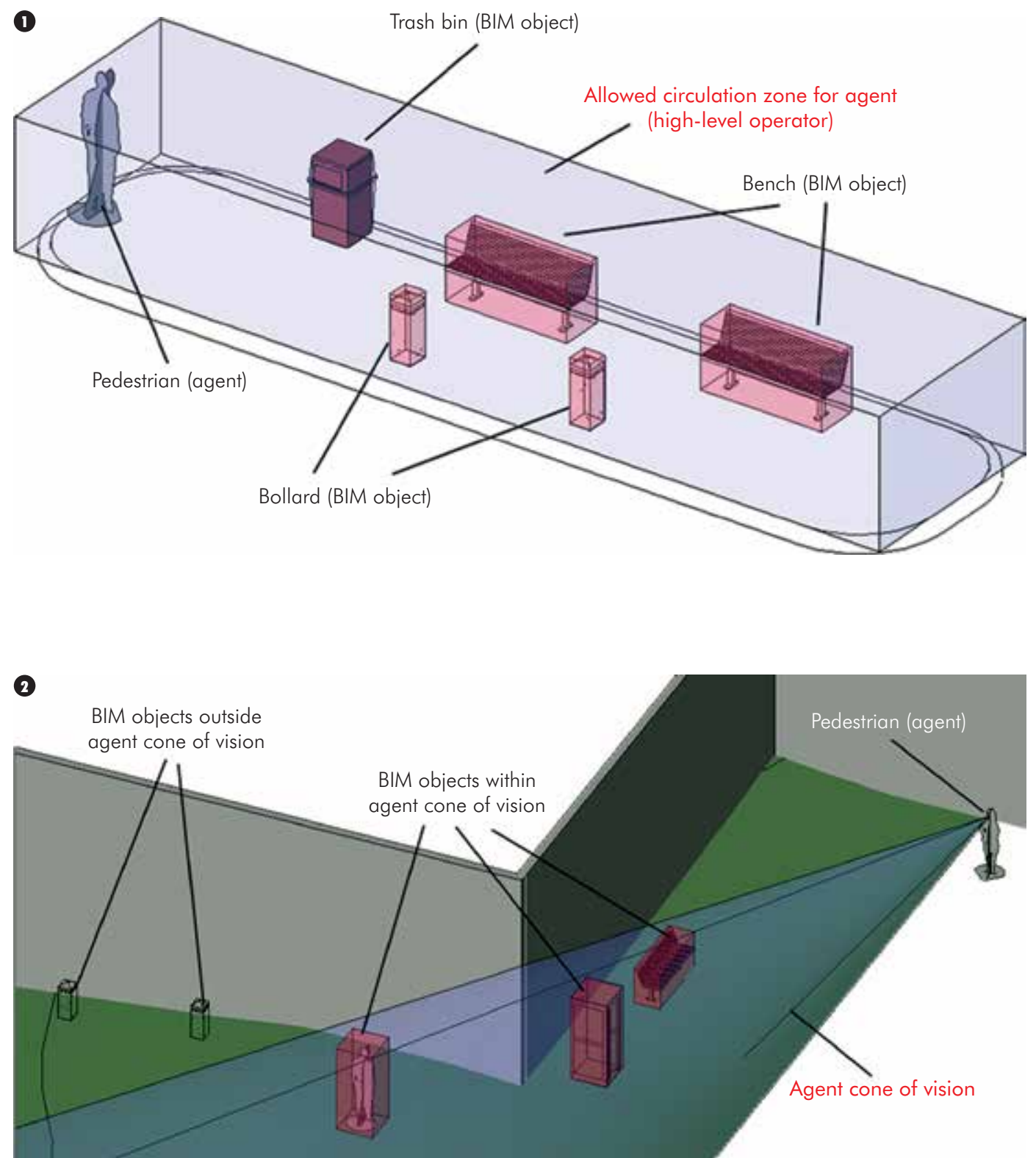

agent. The goal is to build cones of vision for each agent to satisfy rules concerning visual access. BIM objects passed to this operator focus on agents with visual capabilities like pedestrians, bike drivers, and vehicle drivers. Other «visually accessed» objects by these agents are not passed to this operator per se, but are included in other operations that specify intersections between the agent cone of vision and these objects. Arguments passed to this operator include the type of agent, 3D coordinates of agent viewing point, horizontal angle of vision, vertical angle of vision, and the rotation angle of the visual axis.

This operator works as follows: 1) identifying agent type, and extracting associated attributes, 2) locating coordinates of agent viewing point, 
3. Generating «illumination volume» for light post «objects»

3) constructing line of visual axis, normal to plane of sight, 4) constructing profiles of horizontal and vertical cone of vision angles, and 5) revolving profiles around visual axis in 360 degrees to construct cone of vision. This operator applies to rules that mainly deal with agent/agent or agent/object visual Access properties and constraints.

Other high-level operators that can be integrated to satisfy these rules include sweep operations and generating bounding volumes for objects in the scene. 3D low-level operations should also be integrated to achieve results like:

a. Intersecting cones of vision to identify visual communication.

b. Generating common volumes or areas of intersection between sight capabilities of an agent and certain objects to identify visual access.

c. Moving and rotating agents and their cones of vision respectively to obtain desired vision capabilities to check appropriate agent location coordinates.

\section{CONSTRUCT ILLUMINATION VOLUME}

This operator defines a volume (e.g. cone, cylinder) of light caused by an illumination source. These volumes are used to define illuminated areas, areas of lighting overlap, and areas in shade. The main purpose is to identify agents and objects in complete illumination, complete shade or combinations of both. Objects passed to this operator include sources of illumination like lighting poles, vehicle lights, posts, or objects in effect of illumination like roadways, fire hydrants, and pedestrians. Arguments include type of illumination source, source 3D coordinates, direction vector of light, center of spotlight, and type of generated volume of illumination (conical, directional). This operator works as follows: 1) identifying illumination source coordinates, 2) constructing axis of light according to direction vector, 3) constructing light volume profile according to source type, 4) and revolving or sweeping profile around axis to generate illumination volume.

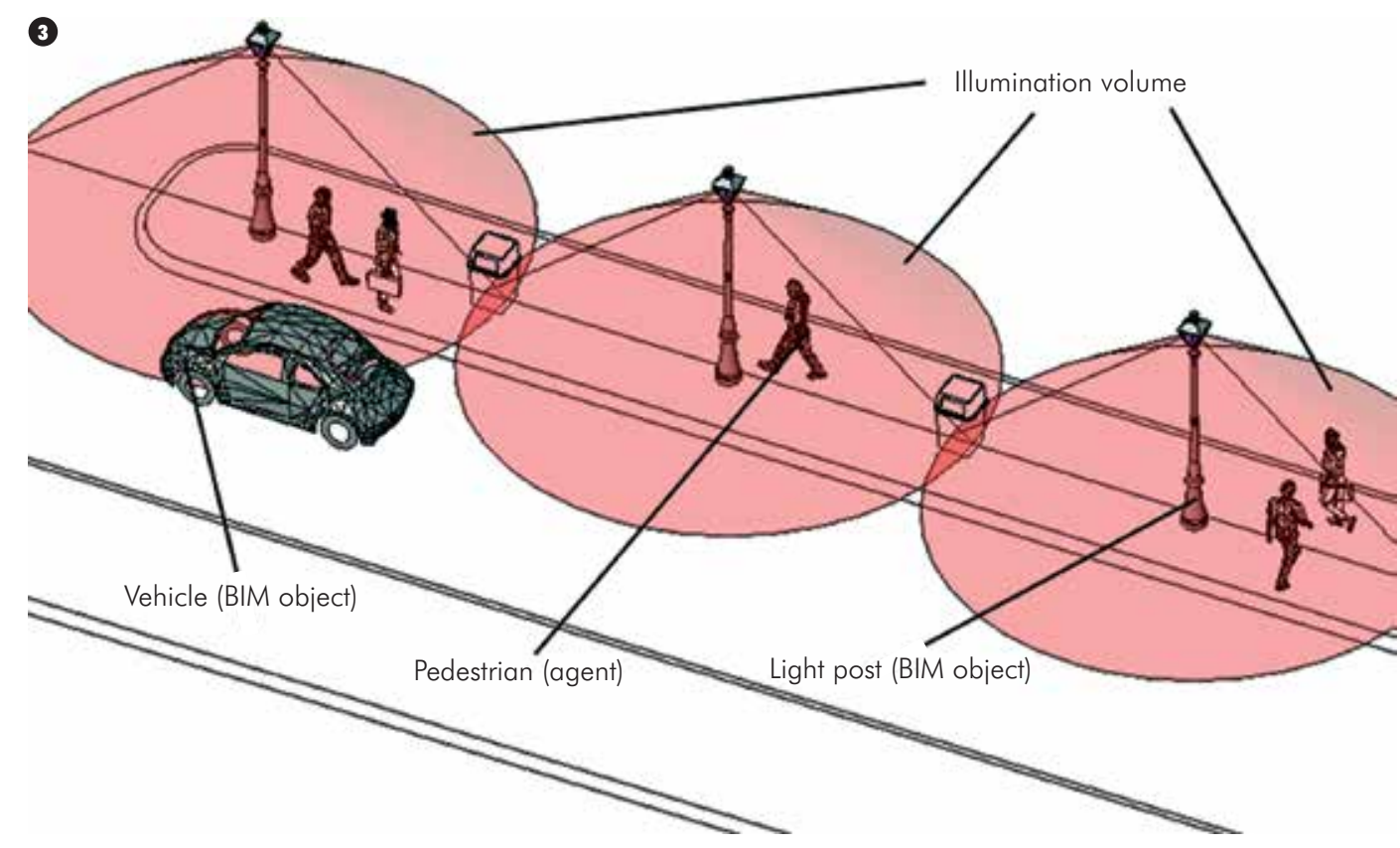

This operator applies to rules that involve identifying lighting and shading areas in circulation paths, checking the location of objects that should or should not lie in illuminated or shaded areas, and checking vehicle night visibility. Other high-level operators include sweep operations, bounding volume operators, and additional 3D operations, mainly intersections of geometrical volumes. 3D lowlevel operations should also be integrated to achieve results like:

a. Identifying regions of overlap of illumination, and computing their volumes and height from ground surface.

b. Identifying regions in shade, defined as the regions outside the set of illuminated regions.

c. Identifying and enumerating objects and agents in illuminated or shaded areas, by generating and counting the solid intersections between object bounding volumes and illumination volumes.

d. Calculating sight distances at night for vehicles, by generating illumination volumes of vehicle light beams, and identifying regions of intersection with vehicle roadways.

\section{DEFINE BOUNDING VOLUME}

This operator defines a volume to bound agents and BIM objects to facilitate the manipulation of associated rules. Defining a bounding volume currently exists in modeling software, but the idea is to use it to explicitly define swept volumes representing motion, direction, and simulation of movement along paths. This operation should be ideally used for agents which are complex to model. For example, it could represent pedestrians, bikes, vehicles and wheelchairs to simulate their movement. Arguments include agent type, maximum dimensions (maximum width, length and height, or radius to distant point), coordinates of agent location around the arbitrary origin, and the desired volume (box, cylinder, sphere.). This operator works as follows: 1) recognizing agent, 2) identifying type of desired bounding volume, 3) constructing profile of bounding region (plane, circle), and 4) generating bounding volume (by sweep, revolving or suitable operation).

Other high-level operators that can be integrated include sweep or revolve operations, and other basic 3D operations that define functions for the 
4. Generating «bounding volume» for «agents» and «objects».

5. Generating «sweep» for pedestrian «agents» and a vehicle «object» along a path.

(
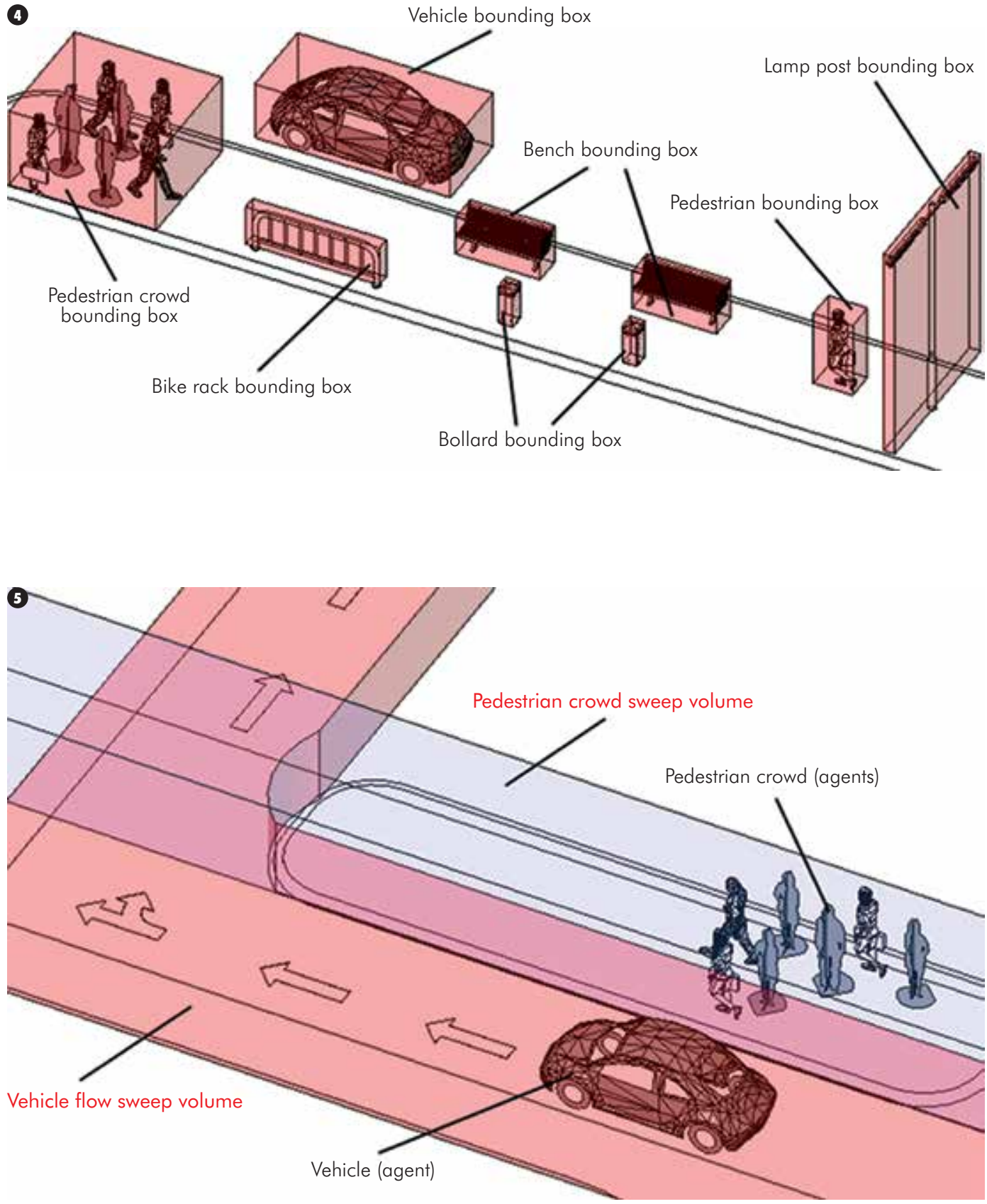

bounding volume (extending it, sweeping it along a path), in addition to basic Boolean operations for identifying geometrical conflicts. High-level operators and 3D low-level operations are usually integrated to achieve results like:

a. Modeling pedestrians to check for visual access.

b. Modeling bikes and vehicles to identify spatial conflicts in roadways.

c. Modeling vehicles to check roadways against suitable visual communication.

\section{SWEEP ALONG PATH}

This operator constructs a sweep of an agent or BIM object along a specific path. The goal of this operator is to generate extrusions of geometric entities along paths to identify properties of spatial conflict, obstructed access, visual communication, and illumination or shading. In most cases, the purpose is to simulate the circulation of involved agents or objects. Arguments include agent or object type, and sweep direction vector. This operator Works as follows: 1) identifying agent/object geometry, coordinates or bounding volume, 2) identifying agent/object faces as individual profiles for sweep operations, and 3) constructing sweeps along desired paths.

This operator applies to most rules that involve objects or agents, where the simulation of their circulation in outdoor space is significant for further purposes. Additional operators consist of both complementary high-level operators and 3D low-level operations like Boolean operations. These are integrated to achieve results like:

a. Simulating routes or mechanisms of fire apparatus to check access conditions to buildings.

b. Simulating vehicle movement to check spatial conflict with elements like bollards. 
6. Proposed mechanism of integration of operators for outdoor circulation rule checking

7. Mechanism of integration of operators for agent-agent / agent-object intersection rule.

\section{INTEGRATION OF OPERATORS TO CHECK RULES}

\section{MECHANISM OF INTEGRATION}

As shown above, multiple high-level operators can be used, individually or combined, to check specific rules. In some cases, high-level operators may not be sufficient to assess the rule. Some operations may be conducted before, between or after the high-level operators to make the checking test complete. The proposed mechanism of integration of operators for rule checking works as shown in FIGURE 6.

This diagram consists of three phases: 1) constructing geometry, 2) 3D editing, and 3) rule testing. The diagram assumes a pool where all geometric models are integrated and operated by 3D low-level operations to check against the corresponding rule(s). These models come from the following sources:

1. Geometric models generated using primary high-level operators: These include allowed circulation zone models, cone of vision models, illumination volume models, bounding volume models, and sweep models. Primary operators refer to operators specific to the rule goal (e.g. cone of vision operator is specific to visual access, all other operators are secondary). There can be one or more primary operators involved to check one rule. These can be manipulated using 3D operations, regardless of any other geometry (intersection between groups of illumination volume models to identify shaded areas).

2. Geometric models generated using secondary high-level operators: These are similar to primary operators but are not the main focus of the rule goal. These operators can be combined with other primary or secondary operators, or a combination of both, to define given checks (combining sweep volume to allowed circulation zone operator to check agent/object intersection).

3. Geometric models from existing geometry: These are BIM objects that are already modeled (bench, lighting pole, pedestrian walkway, etc.). This geometry can be used in

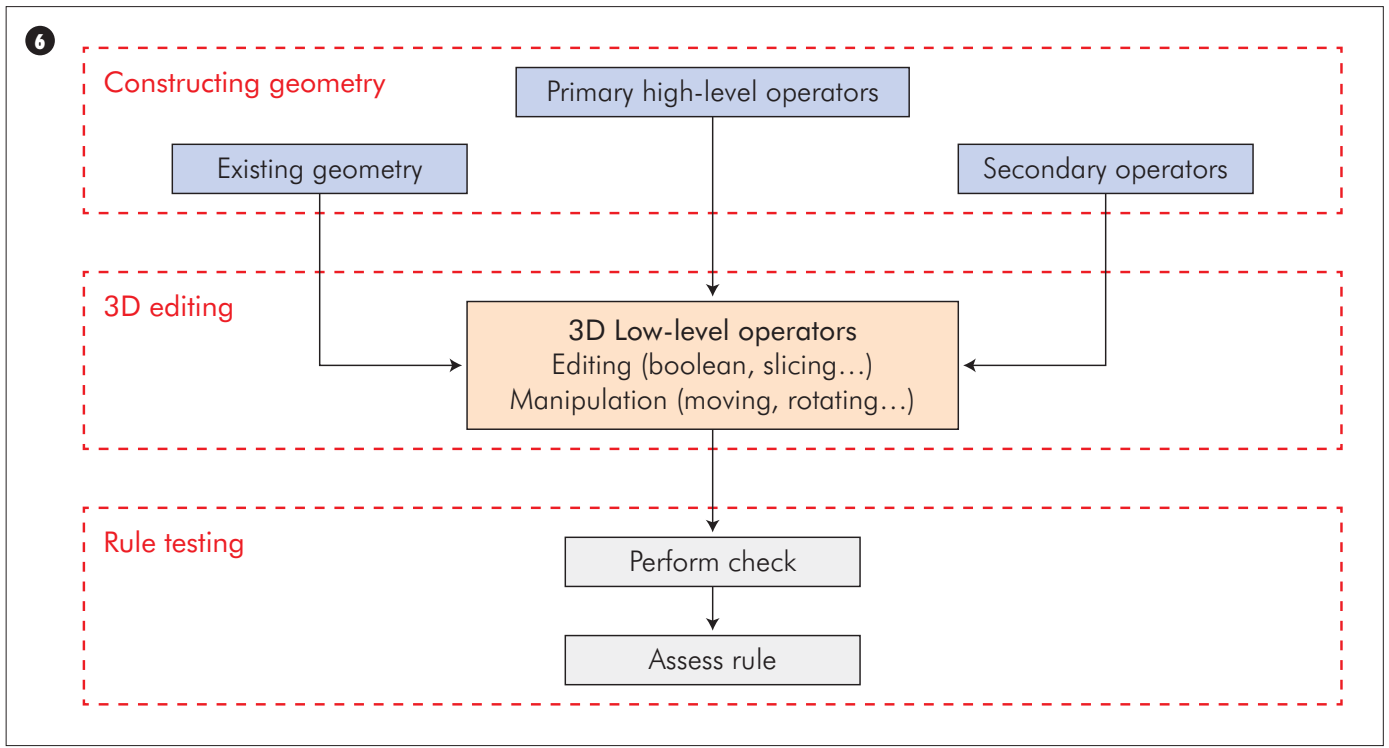

\section{0}

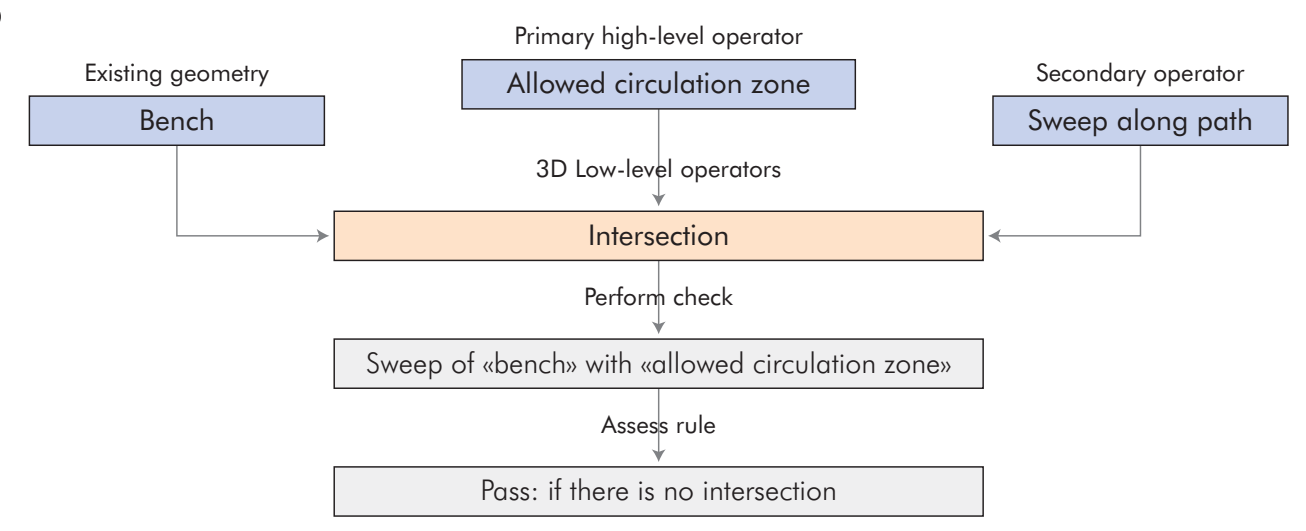

combination with high-level operators to satisfy some rules (intersecting cone of vision models with objects to identify spatial conflicts). Low-level operations (especially intersection operations) are then performed for this pool of models to identify zones of conflict, followed by an evaluation of the operation results.

\section{DOMAINS OF CHECKING}

This section walks through the process of satisfying rule checking requirements within the framework of rule goals described earlier.
CheCKING AGENT-AGENT / AGENT-ObJeCt INTERSECTION RULES

The primary high-level operator for checking these rules is the "construct allowed circulation zone» operator. It involves identifying conflicts of intersection between agents, BIM objects and combinations of both. Consider the rule «Benches should be located so as not to conflict with major pedestrian flow», Ramsey et al. (2000). FIGURE 7 shows the main mechanism for checking this rule. 
The «allowed circulation zone» operator defines the volume of possible pedestrian movement. Both «pedestrian» agents and «bench» objects need to be checked for intersection. If they do not intersect, the check passes; otherwise there is a rule violation. An important caveat is the clear definition of «allowed circulation zone». This defines a volume representing all possible movement routes, but does not exclude other BIM objects like trees, podiums and lighting poles. Disregarding this definition can render the operation inaccurate. Another set of operations needs to be defined in parallel to represent the model correctly. These operations include enumerating all BIM objects that are "allowed» to intersect the path (e.g. trees, podiums) and identifying geometry, and subtracting those objects from the allowable circulation zone model. The resulting volumes represent the actual circulation zone allowed for pedestrian circulation (void where pedestrians can actually walk).

\section{CHECKING AGENT-AGENT / AGENT-OBJECT VISUAL ACCESS} RULES

The primary high-level operator for checking these rules is the «construct cone of vision» operator. It involves identifying conflicts of visual access between agents, BIM objects and combinations of both. Consider the rule «lt is important that sight lines are unobstructed along both roads of an intersection and across their corners for distances sufficient to allow drivers approaching the intersection simultaneously to see each other in time to prevent collision», Harris and Dines (1998). Figure 8 shows the main mechanism for checking this rule.

The "construct cone of vision» operator defines the visual field of a vehicle driver located at specific coordinates in the site. Both the «vehicle driver» agent and the «vehicle» object at the opposite crossroad intersection have to be constructed by the secondary operator "define bounding volume». The cone of vision is constructed from the coordinates of the driver vehicle's viewing location within the bounding box. If the cone of vision intersects with the

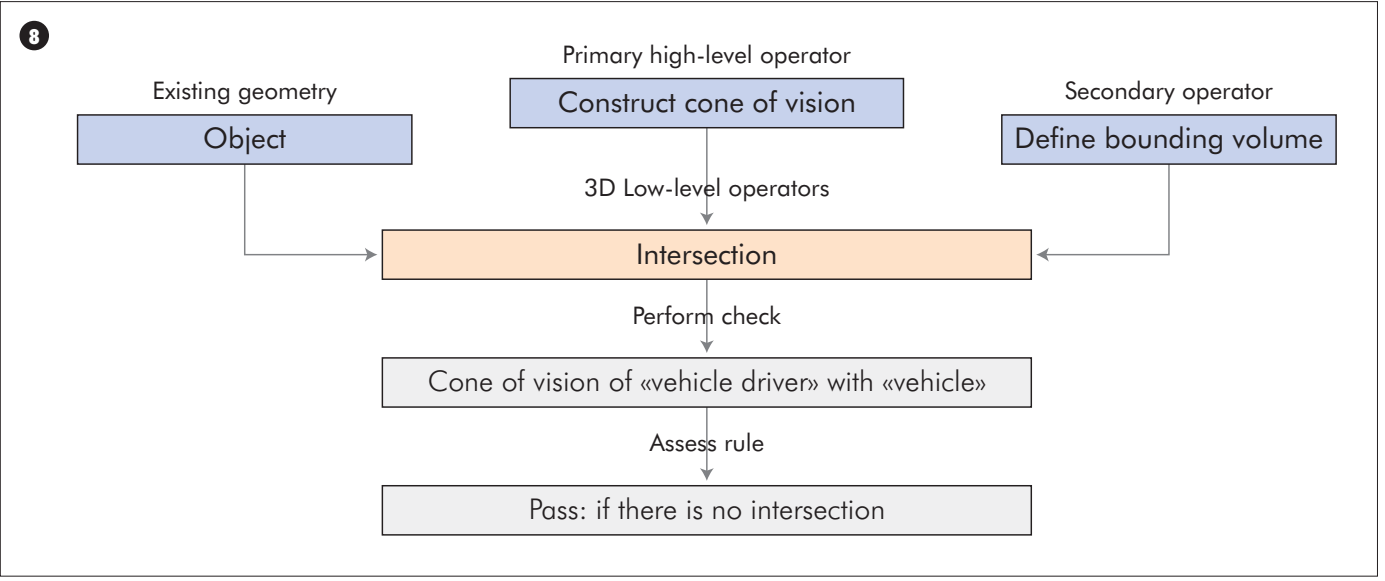

bounding box of the other vehicle, the check passes; otherwise there is a violation.

There are some caveats to this rule. The notion of intersection in geometric modeling is possible even if multiple consecutive intersections occur in front of each other. So if an object intersects the cone of vision before the vehicle, this would not be predicted. Another caveat involves the specific geometry embedded in the rule, i.e. the definition of the cone of vision. Not all of the cone of vision obviously should be included in this rule. To solve this, additional operations need to be defined including slicing the cone of vision according to the exact geometry mentioned in the rule, enumerating all intersections of the cone of vision with any possible objects in the site, and including them in an ordered list (consisting of all intersections). The check passes if the vehicle is the first element in the list.

\section{CHECKING UNOBSTRUCTED ACCESS RULES}

The primary high-level operator for checking these rules is the «sweep along path» operator. It involves identifying conflicts of obstructions for access among objects. Consider the rule «Utility poles can obstruct use of aerial ladders for rescue and fire suppression operations...Canopies and other non-structural building components can also prevent fire apparatus operations close to buildings», Ramsey et al. (2000). FIGURE 9 shows the main mechanism for checking this rule. If the sweep operation of the «aerial ladder» object (which should be defined by a bounding box) intersects with a «utility pole» object, it violates the rule. Normally this check will not be complete unless a "simulation» of the bounding box of the «fire apparatus» occurs, testing against a volume which defines a known spacing distance between utility poles. This should lead to more accurate checking.

CHECKING OUTDOOR LIGHTING AND SHADING RULES

The primary high-level operator for checking these rules is the "construct illumination volume» operator. It involves identifying conflicts of volumes of shade and light among objects. Consider the rule: «Streets that are properly lighted enable fire fighters to locate hydrants quickly and to position apparatus at night. Avoid layouts that place hydrants and standpipe connections in shadows», Ramsey et al. (2000). FIGURE 10 shows the main mechanism for checking this rule.

If the «illumination volume» object intersects with the «fire hydrant» object, the check passes; otherwise it violates the rule. A key issue is multiple intersections of illumination, which implies a probability of being in shade while the illumination volume intersects. This would require constructing an ordered list of all possible intersections with the illumination volume. The check passes if the fire hydrant was first in order. 
9. Mechanism of integration of operators for unobstructed access rule.

10. Mechanism of integration of operators for outdoor lighting and shading rule.

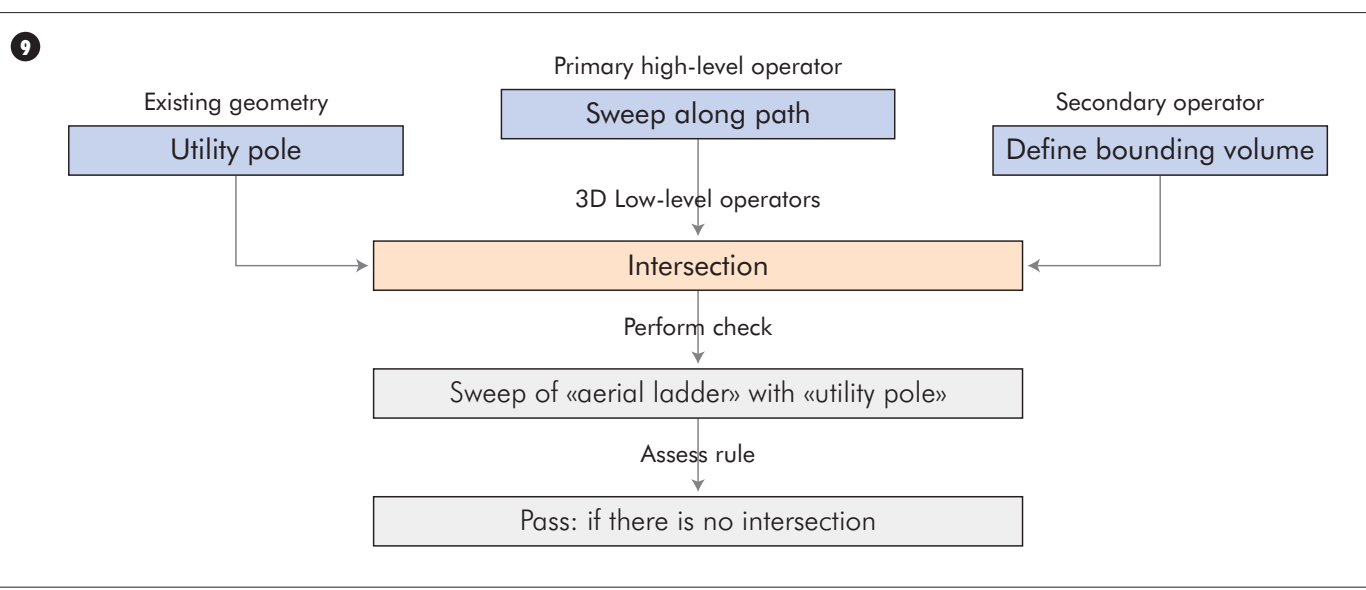

(1)

Existing geometry Fire hydrant, lighting pole
Primary high-level operator Construct illumination volume

\begin{tabular}{c} 
3D Low-level operators \\
Intersection \\
"Illumination volume» with «fire hydrant» \\
Assess rule \\
Pass: if there is no intersection \\
\hline
\end{tabular}

Secondary operator Define bounding volume

\section{DISCUSSION}

One of the key issues pointed out in the type of rule checking outlined in this paper is the idea of simulating or modeling behavior of certain agents or objects. This does not necessarily imply using simulation-based rule checking as a method, but involves the potential of using geometric modeling principles to address situations where text-based checking cannot identify alone. One of the main observations is that most operations go downstream to a group of 3D low-level operations involving intersection. This may be due to the fact that most rules deal with visual and spatial conflicts or obstructions. It was observed, however, that some rules required other types of operations (e.g. slicing, subtracting) to identify additional properties. These were not intrinsic to the nature of the rules, but to geometrical modeling capabilities. Inspite of the attempts to fill in caveats in some rules that needed more than just definitions of high-level operators, there can still be a lot of «unknown» error checks, which cannot be easily identified by these operators. A closer look at the interaction of operators is required to develop a sound set of operators and underlying operations.

Regarding the area of outdoor circulation, there is probably a lot more to explore. It is still an area with a very few attempts in rule checking. What can or cannot be checked at this point according to the geometrical approach is a significant issue which can lead to further research. Some rules cannot be currently implemented, especially those involving speed, density, flow, and efficiency. There are areas other than circulation that need to be explored, such as landscape terrain and topography, water drainage and other issues which can potentially be implemented through a geometric modeling approach. An important constraint however is IFC support for the domain of outdoor circulation. IFC extensions are still limited to indoor space planning, circulation, and area calculation issues. The definition of outdoor entities such as walkways, roadways, fences, gates, trees, and benches and other objects, and providing suitable geometric capabilities for manipulating such entities could be a push toward a new field of future research.

The integration between BIM (in the form of IFC entities) and GIS (in the form of e.g. CityGML types and entities) is one of the promising areas of research in this regard.

\section{REFERENCES}

Abbey, B. (1998). U.S. landscape ordinances: an Annotated Reference Handbook, New York: John Wiley and Sons Australia Limited.

Ding, L., Drogmuller, R., Jupp, J.R., Rosenman, M. and Gero, J.S. (2004). Automated Code Checking, International CRC Construction Innovation Conference.

Eastman, C., Lee, J.-K., Sheward, H., Sanguinetti, P., Jeong, Y.-S., Lee, J. and Abdelmohsen, S. (2009). Automated Assessment of Early Concept Designs, in Architectural Design, Vol. 79 NN$^{\circ}$ 2: 52-57, DOI: 10.1002/ad.851.

Harris, C. and Dines, N. (1998). Time-saver standards for landscape architecture: design and construction data, McGraw-Hill Professional.

Hillier, B., Penn, A., Hanson, J., Grajewski, T., and Xu, J. (1993). Natural movement: or configuration and attraction in urban pedestrian movement, Environment \& Planning B: Planning \& Design, volume 19, 29-66.

Hoogendoorn, S. (2003). Pedestrian Travel Behavior Modeling, Proceedings of the $10^{\text {th }}$ International Conference on Travel Behavior Research, Lucerne. 
Kerridge, J., Hine, J., and Wigan, M. (2001). AgentBased Modelling Of Pedestrian Movements: The Questions That Need To Be Asked and Answered. Environment and Planning B: Planning and Design. Vol. 28, pp. 327-341.

Landphair, H. and Klatt, F. (1999). Landscape architecture construction, $3^{\text {rd }}$ ed., Upper Saddle River, N.J.: Prentice Hall PTR.
Lee, J.-K., Eastman, C., Lee, J., Kannala, M. and Jeong, Y.-S. (2010). Computing walking distances within buildings using the universal circulation network, Environment and Planning B: Planning and Design, 37(4) 628-645.

Raford, N. and Ragland, D. (2003). Space Syntax: An innovative pedestrian volume modeling tool for pedestrian safety. Paper 04-2977, Annual Meeting of the Transportation Review Board.
Ramsey, C., Sleeper, H. and American Institute of Architects (2000). Architectural Graphic Standards, John Ray Hoke, Jr., editor in chief, $10^{\text {th }}$ ed., New York: John Wiley \& Sons.

Sanguinetti, P., Abdelmohsen, S., Lee, J.M., Lee, J.K. Sheward, H. and Eastman, C. (2012). General System Architecture for BIM: An Integrated Approach for Design and Analysis, Advanced Engineering Informatics, Vol. 26, Issue 2, 317 333. 